\title{
Can Parasites Ameliorate or Prevent IBD and other Immune- mediated Diseases?
}

\author{
Robert W Summers \\ Department of Internal Medicine, Division of Gastroenterology and Hepatology, \\ University of Iowa Carver College of Medicine, USA
}

See the article pages: 96-104

\section{Background}

The pathogenesis of inflammatory bowel disease appears to involve a dysregulated and destructive immune response to intestinal contents. The primary etiology remains unknown, but both genetic and environmental factors are likely to play a role. Crohn's disease and ulcerative colitis both occur in increased frequency in some families and numerous susceptibility genes are involved. However, it appears that the application of highly hygienic practices have been associated with the progressive increase of IBD prevalence and other immune mediated disease in many Western industrialized countries [1]. As parasites are being eradicated by rigorous hygienic practices and specific treatment, immune diseases are increasing dramatically around the world. These diseases include multiple sclerosis, psoriasis, allergic and asthmatic disorders and even type I diabetes mellitus [2]. Parasites coexist with their hosts through modification of their host's immune system. It has been hypothesized that the emergence of inflammatory bowel disease and other immune disorders occurs because of the loss of exposure to helminths and their resulting modulation of the immune system. This is being called the helminth hygiene hypothesis. Weinstock, Elliott and numerous other investigators have elucidated many of the helminth-induced immune regulatory pathways that suppress destructive and inappropriate intestinal inflammation[3].

Furthermore, several early clinical trials have suggested that introduction of helminths, such as Trichuris suis and Necator americanus, to persons with inflammatory bowel disease produces improvement in disease activity [4-7]. Additional clinical trials are in progress and others are planned to further investigate whether such therapy is safe and effective.

\section{Summary of paper}

The paper entitled "Impact of treatment of Intestinal parasites on the activity of Ulcerative colitis" approaches the IBD/parasite interaction by removing pre-existing parasites from ulcerative colitis patients instead of introducing them as therapy. The authors enrolled 20 patients with ulcerative colitis who had intestinal parasites. After baseline studies, specific antiparasitic therapy was given to ten of them, and the other ten remained untreated. All were evaluated in one month. In treated subjects, parameters deteriorated or remained unchanged while untreated patients they remained the same or improved. The results imply that removing the parasites was harmful and support the concept that the immune system in ulcerative colitis was adversely affected in the absence of parasites.

\section{Comment on the study}

Withdrawal of a potentially beneficial treatment is a valid and innovative design of clinical trials. Unfortunately, the number of patients in this study was too small to achieve statistical significance, but the results support a beneficial role of helminth-induced immunomodulation in an immune mediated disease. It is surprising that patients with single cellular protozoa such as Entamoeba histolytica, Giardia lamblia and Blastocystis hominis were included in the study, but removing them also seemed to have adverse effects on the colitis. Helminths exert multiple mechanisms in their host including induction of Th2 immune responses and directing immune responses away from Th1/Th17. They also induce production of IL-4, IL-10 and IL13 and inhibit IL-12 and TNF- $\alpha$ release. Perhaps the most important mechanism against immune mediated injury is promotion of regulatory circuits [8-9]. Eukaryotic protozoan pathogens also have evolved to evade immune defenses responses of their host, but through entirely 
different mechanisms. A review describes how protozoa avoid immune attack by using humoral effector mechanisms through resistance of complement lysis, resistance to intracellular lysosomal enzymes and toxic metabolites and modifying antigen-presenting and immunoregulatory functions of dendritic cells [10]. There is almost no clinical or experimental evidence to support their role in ameliorating immune mediated disease. On the other hand, until their effects on immune mediated diseases are explored in more detail, their ability to ameliorate destructive immune and inflammatory processes remains possible. On the other hand, evidence supporting the use of helminths in immune mediated diseases is abundant in epidemiological studies, experimental animal disease models and early clinical trials [11-12].

\section{Recommendations}

The current study is interesting, innovative, and provocative. The effects of treating and abolishing parasites should be further explored in ulcerative colitis and other immune mediated diseases. Because the ways in which these two parasites evade the immune system of the host are quite different, helminths and protozoa should be investigated in separate cohorts and treatments should be administered using a double blind, placebo-controlled design. As immunemediated diseases increase throughout the world, it is increasingly urgent to find measures to prevent and treat them. Re-introduction of old companions shows promise to provide relief.

\section{References}

1. Weinstock JV, Summers R, Elliott DE. Helminths and harmony. Gut 2004; 53:7-9.

2. Bufford JD, Gern JE. The hygiene hypothesis revisited. Immunol Allergy Clin North Am 2005; 25: 247-62.
3. Weinstock JV, Elliott DE. Helminths and the IBD hygiene hypothesis. Inflamm Bowel Dis. 2009; 15:128-33.

4. Mortimer K, Brown A, Feary J, Jagger C, Lewis $\mathrm{S}$, Antoniak $\mathrm{M}$, et al. Dose-ranging study for trials of therapeutic infection with Necator americanus in humans. Am. J. Trop. Med. Hyg. 2006; 75:914-20.

5. Croese J, O'neil J, Masson J, Cooke S, Melrose W, Pritchard D, Speare R. A proof of concept study establishing Necator americanus in Crohn's patients and reservoir donors. Gut 2006; 55:1367.

6. Summers RW, Elliott DE, Urban JF, Thompson $\mathrm{R}$, Weinstock JV. Trichuris suis therapy in Crohn's disease. Gut 2005; 54:87-90.

7. Summers RW, Elliott DE, Urban JF, Thompson RA, Weinstock JV. Trichuris suis therapy for active ulcerative colitis: a randomized controlled trial. Gastroenterology 2005; 128:825-32.

8. Elliott DE, Weinstock JV. Helminth-host immunological interactions: prevention and control of immune-mediated diseases. Ann. N.Y. Acad. Sci. 2012; 1247:83-96

9. McSorley HJ, Hewitson, JP, Maizels RM. Immunomodulation by helminth parasites: Defining mechanisms and mediators. Int $J$ Parasit 2013; 43:301-310.

10. Sacks D, Sher A. Review: Evasion of innate immunity by parasitic protozoa. Nature Immunol 2002; 3:1041-47.

11. Chacin-Bonilla L. Relevance of helminths in the prevention and healing of immune diseases. Invest Clin 2009; 50:1-4. (Spanish)

12. Elliott DE, Weinstock JV. Helminthic therapy: using worms to treat immune-mediated disease. Adv Exp Med Biol. 2009; 666:157-66 